\title{
Tetramethylguanidine-based gels and colloids of cellulose
}

\author{
Gonçalo V.S.M. Carrera ${ }^{\mathrm{a}, *}$, Anabela Raymundo ${ }^{\mathrm{b}}$, Francisco M. Braz Fernandes ${ }^{\mathrm{c}}$, \\ Noémi Jordão ${ }^{a}$, Isabel Sousa ${ }^{\mathrm{b}}$, Manuel Nunes da Ponte ${ }^{\mathrm{a}}$, Luís C. Branco ${ }^{\mathrm{a}, *}$ \\ a LAQV/REQUIMTE Faculdade de Ciências e Tecnologia, Universidade Nova de Lisboa, CQFB/REQUIMTE, 2829-516 Caparica, Portugal \\ ${ }^{\mathrm{b}}$ Instituto Superior de Agronomia, Universidade de Lisboa, LEAF - Linking Landscape Environment Agriculture and Food, Tapada da Ajuda, $1349-017$ \\ Lisboa, Portugal

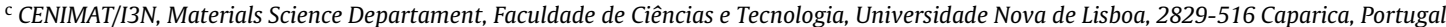

\section{A R T I C L E I N F O}

\section{Article history:}

Received 17 October 2016

Received in revised form 13 March 2017

Accepted 27 March 2017

Available online 29 March 2017

\section{Keywords:}

Cellulose

Gels

Colloids

NMR

Rheology

Molecular interactions

\begin{abstract}
A B S T R A C T
Novel and stable gels of cellulose were produced. These gels are prepared at room temperature by combination of cellulose and tetramethylguanidine (TMG) in different ratios $(1: 1,1: 2,1: 3$ in equivalents of alcohol groups of cellulose per number of molecules of TMG). Detailed NMR, SEM, rheological and XRD studies of these gels were carried out. The concentration of cellulose in the gel, temperature, frequency of oscillation and shear rate were used as variables in order to understand the fundamentals and optimize operational conditions, considering their possible use as matrices for $\mathrm{CO}_{2}$ capture. Cellulose recovery from a specific gel was performed using ethanol as precipitating agent, leading to a lower crystallinity, which permits to consider this polymer in further studies associated to physical/chemical modification of cellulose.
\end{abstract}

(c) 2017 Elsevier Ltd. All rights reserved.

\section{Introduction}

Cellulose, the most abundant organic compound on earth obtained from renewable resources, has enormous applications in the pharmaceutical and fine chemical industries, in the development of new materials and as an alternative energy resource. Nevertheless, the processing of this polysaccharide is a challenge due to the strong intra- and intermolecular hydrogen-bonds between the different - $\mathrm{OH}$ groups present in the polymeric chain, which precludes an effective solubilisation in most solvents.

We found in our labs, initially by serendipity, that cellulose forms homogeneous gels using a single component, commercially available and cheap tetramethylguanidine (TMG), an organic superbase.

In fact, other authors tested systems containing organic superbases in the context of cellulose solubilization. As examples (King, Asikkala, Mutikainen, Järvi \& Kilpeläinen, 2011) tested diverse protic ionic liquids, with basic anions, in order to obtain fast dissolution of moderated quantities of cellulose at high temperatures and, with

\footnotetext{
* Corresponding authors.

E-mail addresses: goncalo.carrera@fct.unl.pt (G.V.S.M. Carrera), anabraymundo@isa.ulisboa.pt (A. Raymundo), fbf@fct.unl.pt (F.M.B. Fernandes), noemicjordao@gmail.com (N. Jordão), sabelsousa@isa.ulisboa.pt (I. Sousa), mnponte@fct.unl.pt (M.N. da Ponte), l.branco@fct.unl.pt (L.C. Branco).
}

a judicious choice of anion and temperature, control the degree of polymerization of cellulose as well as the recyclability of the system. Additionally, the authors highlight the importance of basic moieties able to disrupt, by hydrogen bond, the strong intra- and inter-molecular interactions among cellulose polymeric chains. A different system (Wang, Xue, Yan, Li \& Mu, 2016) consists on the use of an organic superbase in DMSO to solubilize cellulose under an atmosphere of $\mathrm{CO}_{2}$. The concept follows the principle of reversible ionic liquid/molten salt (with $\mathrm{CO}_{2}$ as element of reversibility) previously applied in our labs. In concrete, we have reported the use of organic superbases, such as TMG and DBU, in combination with amines, aminoacids, mono- and oligosaccharides, as reversible $\mathrm{CO}_{2}$ capture systems (Carrera, Jordão, Branco \& Nunes da Ponte, 2015a,b; Carrera, Nunes da Ponte \& Branco, 2012; Carrera, Jordão, Santos, Nunes da Ponte \& Branco, 2015).

In this context, and in order to capitalize the very high values of cellulose uptake by TMG, we carried out a detailed spectroscopic, rheological, SEM and XRD evaluation. Such studies are essential to understand the intermolecular interactions ruling the Cellulose/TMG system, as well as to find optimized operational conditions to use these mixtures as films and membranes for $\mathrm{CO}_{2}$ capture. Other possible applications reported in the literature consist in novel material preparation, (Frey, Chan \& Carranco, 2005) or chemical, (Zhou, Zhang, Deng \& Wu, 2004) and physical (Gavillon 


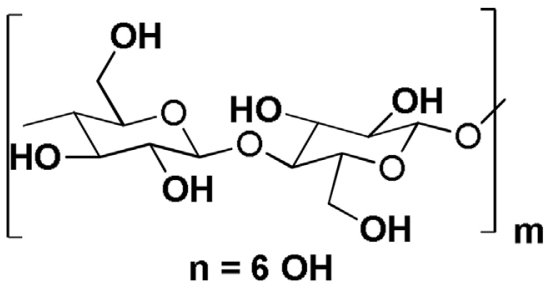

Cellulose<smiles>CN(C)C(=N)N(C)C</smiles>

$0=m \times(n$ or $2 n$ or $3 n)$

Tetramethylguanidine

Fig. 1. Components of the prepared gels: cellulose and tetramethylguanidine.

\& Budtova, 2007; Xia, Patchan, Maranchi \& Trexler, 2015) modification of this polymer.

From the diverse cellulose/solvent systems, the concept of gel of cellulose is thoroughly studied, mostly in aqueous-based systems and different combinations of additives. As an example, it is important to highlight the work (Roy, Budtova \& Navard, 2003) focused on the rheological properties and gelation of aqueous/NaOH/cellulose solutions, where the temperature effect on aggregation of cellulose chains, separation from solvent matrix and irreversibility of the gels were reported. Those found contrast with methylcellulose hydrogel-based properties, (Li et al., 2001) with intermediate degree of substitution of the hydroxyl groups by methoxides, where no additive is required for solubilisation in water and reversibility of gelification. Additional studies were carried out using water/NaOH as solvent, with inclusion of additives such as thiourea (Ruan, Lue \& Zhang 2008) and urea, (Cai \& Zhang 2006) presenting similarly an irreversible behaviour on gelification with variation of temperature. The inverse relation between concentration of cellulose and temperature of gelification is stated in these studies to be a strong indication that, at higher concentrations of this polymer, lower temperatures are required to promote disruption of hydrogen bond-based interactions between the solvent system and cellulose, and the consequent entanglement between the different polymer chains, leading to aggregation and formation of a gel.

Different kinds of rheological measures on cellulose-based gels can give valuable structural insights, such as $\mathrm{G}^{\prime}$ (storage modulus), which represents the elastic behavior of the material, as a measure of the deformation energy stored during the shear process, and $\mathrm{G}^{\prime \prime}$ (loss modulus), the viscous component of the material, a measure of the energy used to flow, and therefore lost, during the shear process. (Angioloni \& Collar, 2009) Both parameters are measured as functions of stress, frequency and temperature, stimuli that promote or disrupt phase separation between solvent matrix and cellulose chains as well as aggregation between polymer chains. Another relevant parameter is the flow behaviour in steady shear tests, (Burchard, 2001; Lue \& Zhang, 2009; Zhang, Ding, Huang, Chen \& Yang, 2014) (viscosity $\eta$ ) as a function of temperature and shear rate. A more complete picture of the structural organization of these gels, responsible for a specific behavior, can be given by spectroscopic characterization, in order to aid in the interpretation of results, it is important to highlight the work of (Vitorino et al., 2014), where the X-ray structure of tetramethylguanidinium nitrate and the hydrogen bond-based interactions between the different motifs are reported. The preparation conditions of the mixtures are another important parameter, where period and rate of stirring are essential to obtain an effective mixing between the intervening components. (Qin, Lu \& Zhang, 2012) These studies support some of the experimental results hereby presented. The simplicity of the preparation of these gels, the low associated cost and the potential applications are characteristics that convert these gels in systems worth to investigate from a fundamental point of view.

\section{Materials and methods}

\subsection{Materials}

1,1,3,3 Tetramethylguanidine (TMG) was supplied from SigmaAldrich (99\%) and water was removed using molecular sieves. Microcrystalline cellulose was purchased from Alfa-Aesar (Degree of polymerization <350) and Ethanol (Carlo Erba, p.a.).

\subsection{Methods}

\subsubsection{Preparation of gels}

The gels were prepared by adding $3 \mathrm{~g}$ of TMG to the proper amount of cellulose, $0.465 \mathrm{~g}, 0.698 \mathrm{~g}$, and $1.395 \mathrm{~g}$, corresponding to $1: 3,1: 2$ and $1: 1$ Cellulose:TMG ratios in equivalents of alcohol groups per number of molecules of TMG, respectively, and continuously stirring the mixtures for approximately $48 \mathrm{~h}$ at the rotational speed of $750 \mathrm{rpm}$. After completion of the mixing period, the samples were stored in a freezer.

\subsubsection{Thermal ${ }^{1} H$ NMR spectra}

The prepared gels were characterized, from 25 to $100^{\circ} \mathrm{C}$, by thermal ${ }^{1} \mathrm{H}$ NMR spectra (in a capillary inside an NMR tube containing $\mathrm{d}_{6}$-DMSO as the reference solvent), recorded on a Bruker AMX400 spectrometer. Chemical shifts are reported downfield in parts per million from a tetramethylsilane reference.

\subsubsection{Rheological characterization}

Small amplitude oscillatory system (SAOS) measurements of storage $\left(G^{\prime}\right)$ and loss $\left(G^{\prime \prime}\right)$ moduli were performed in a controlledstress rheometer (Haake, Mars III Germany), using a serrated parallel plate geometry (PP20 $\mathrm{Ti}-20 \mathrm{~mm}$ diameter) with $0.3 \mathrm{~mm}$ of gap. Temperature was controlled by an EHEIM circulating water bath (Germany) coupled with a Peltier system. Thermal study was carried out on three consecutive steps: heating $\left(5-90^{\circ} \mathrm{C}\right)$ in $2700 \mathrm{~s}$, cooling $\left(90-5^{\circ} \mathrm{C}\right)$ in the same period of time and maturation $\left(5^{\circ} \mathrm{C}\right)$ during 1800s. Stresses of 100,10 and $0.1 \mathrm{~Pa}$, under the linear viscoelastic region, were considered after carrying out the respective stress sweeps from the highest to the lowest concentration of cellulose. The oscillation frequency considered was $1 \mathrm{~Hz}$ in all the situations.

Another study was carried out using oscillation frequency as the variable $(0.001-100 \mathrm{~Hz})$ in five steps at $5{ }^{\circ} \mathrm{C}$, considering the same stress values used in the thermal studies.

Moreover, two different steady shear flow studies were performed. The first considering shear rate as a variable $\left(1 \times 10^{-8} \mathrm{~s}^{-1}-500 \mathrm{~s}^{-1}\right)$ in $660 \mathrm{~s}$ (20 steps) at $20^{\circ} \mathrm{C}$ with a serrated parallel plate geometry PP20 Ti $(20 \mathrm{~mm}$ diameter) with $0.3 \mathrm{~mm}$ of gap. In the second study the temperature was considered as the variable: heating $\left(5^{\circ} \mathrm{C}-90^{\circ} \mathrm{C}\right)$ in $600 \mathrm{~s}$ (10 steps), considering a constant shear rate of $1 \mathrm{~s}^{-1}$ using the same geometry and gap. 


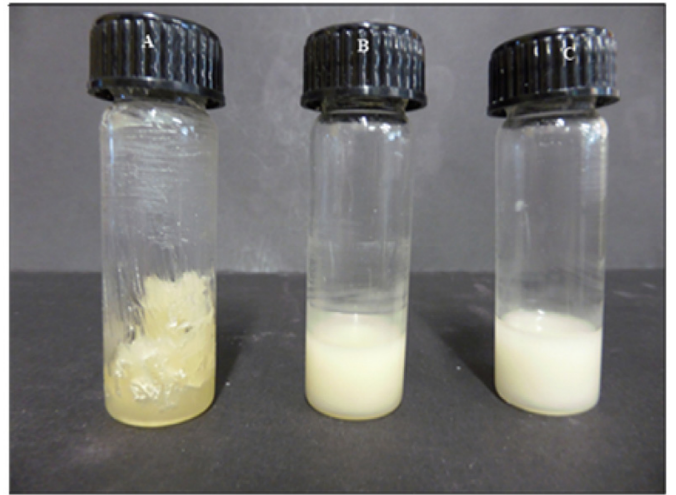

Fig. 2. A, B and C - Gels of Celulose/TMG $-1: 1,1: 2$ and $1: 3$ respectively.

\subsubsection{Isolation of cellulose from the gel using ethanol}

The gel $(0.25 \mathrm{~g}) 1: 2$ cellulose/TMG (in equivalents) was mixed with $3.5 \mathrm{~mL}$ of ethanol, under continuous stirring during $116 \mathrm{~h}$. After that period, the mixture was cooled using ice, and a white solid precipitated. This solid was separated from the alcoholic mixture, and subject to vacuum.

\subsection{5. ${ }^{13}$ C-CPMAS-NMR}

Solid state ${ }^{13} \mathrm{C}$-CPMAS spectra were acquired with a $7 \mathrm{~T}$ $(300 \mathrm{MHz})$ AVANCE III Bruker spectrometer operating at $75 \mathrm{MHz}$ equipped with a BBO probehead. The samples were spun at the magic angle at a frequency of $10 \mathrm{kHz}$ in $4 \mathrm{~mm}$-diameter rotors at room temperature. The ${ }^{13} \mathrm{C}$-CPMAS NMR experiments were acquired with a contact time of $1.2 \mathrm{~ms}$, and the recycle delay was $2.0 \mathrm{~s}$.

\subsubsection{FEG-SEM}

The samples were deposited on a double face carbon tape and then coated with an $\mathrm{Au} / \mathrm{Pd}$ film with ca $30 \mathrm{~nm}$ thickness in a sputter coater Quorum Technologies model Q150T ES.

The prepared samples were visualized in a JSM7001F fieldemission scanning electron microscope (JEOL, Japan).

\subsubsection{X-ray diffraction (XRD)}

Samples were analysed using a Rigaku diffractometer DMAX-III $C$ with a copper sealed tube, excited $40 \mathrm{kV} / 30 \mathrm{~mA}$.

\section{Results and discussion}

The studies here reported comprise the preparation of gels of cellulose using TMG (an organic superbase) as solvent matrix. Different Cellulose/TMG ratios were considered: $1 / 1,1 / 2$ and $1 / 3$ (in equivalents of alcohol groups of cellulose per number of molecules of TMG - Fig. 1).

All the mixtures were stirred for approximately $48 \mathrm{~h}$, until a homogeneous gel was evident, in line with the results presented in the study of (Qin et al., 2012), where the importance of stirring conditions (higher rates, longer periods of mixing) on the efficiency of mixing of the cellulose aqueous solution with $\mathrm{NaOH} /$ urea additives is highlighted. The prepared gels (1:1,1:2 and $1: 3$ eq. Cel:TMG in equivalents Fig. 2A, B and C respectively) correspond to $46.5,23.3$ and $15.5 \mathrm{wt} \%$ of cellulose uptake in TMG, respectively. As far as we know, these are some of the highest values of microcrystalline cellulose uptake that were obtained at room temperature, in contrast with most conventional methods. (Wang, Gurau \& Rogers, 2012)

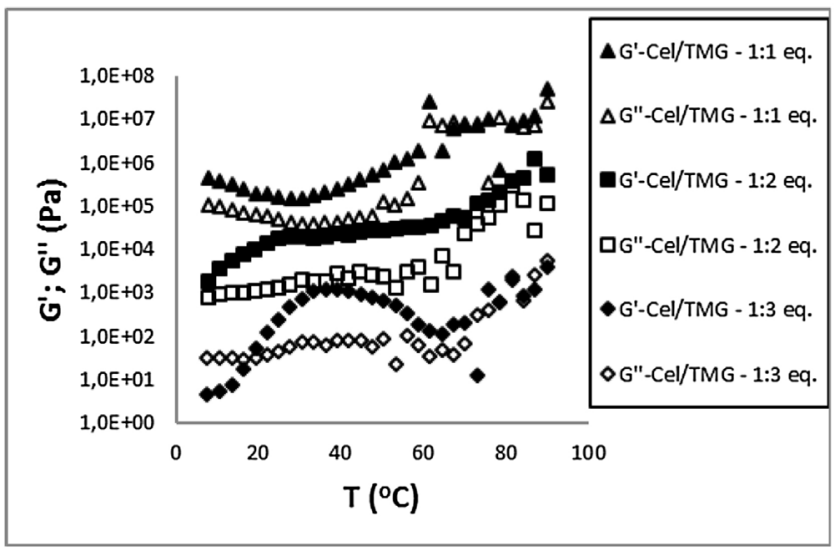

Fig. 3. Heating curves in dynamic rheology measurements with $\mathrm{G}^{\prime}$ (storage) and $\mathrm{G}^{\prime \prime}$ (loss) moduli vs $\mathrm{T}$ (temperature), $\mathrm{f}=1 \mathrm{~Hz}$ for all concentrations of cellulose (Cel) in tetramethylguanidine (TMG) as solvent matrix. In the linear viscoelastic regime: $\tau=0.1 \mathrm{~Pa}$ (Cel/TMG: $1 / 3$ eq.), $\tau=10 \mathrm{~Pa}$ (Cel/TMG: $1 / 2$ eq.), $\tau=100 \mathrm{~Pa}$ (Cel/TMG: $1 / 1$ eq.).

\subsection{Rheological characterization}

The rheological thermal profile ( $\mathrm{G}^{\prime}$ - storage and $\mathrm{G}^{\prime \prime}$ - loss moduli vs temperature - Fig. 3) was obtained in the linear viscoelastic region considering the range $\left(5-90^{\circ} \mathrm{C}\right)$.

A gel can be defined as a continuous network immersed in a liquid medium and exhibiting no steady-state flow. The liquid prevents the network from collapse into a compact mass and the network prevents the liquid from flowing away. (Tanaka, 1981). This implies an internal structure formed by all sorts of intermolecular linkages, including hydrogen-bonding and hydrophobic interactions. The gelling point can be determined by rheology tools when, in the linear viscoelastic regime, storage modulus $\left(G^{\prime}\right)$ surpasses loss modulus ( $G^{\prime \prime}$ ) (Chambon \& Winter, 1987).

During heating, the formation of a gel was registered at different temperatures for different cellulose concentrations in TMG solvent - the fact that the temperature of the crossing point decreases with polymer concentration is a characteristic of a true gel (Ferry, 1980).

In order to understand the behavior presented in Fig. 3, it is important to highlight a work, (Ruan et al., 2008) where a similar study was carried out with cellulose $\mathrm{NaOH} /$ thiourea aqueous solutions, and the progressive structuration from solutions to gels with an increase of temperature is explained by an entanglement of cellulose chains creating a network. From Fig. 3, it can also be noticed that the degree of structuration increases with polymer concentration, as the magnitude of the viscoelastic parameters increase.

Moreover, irreversibility was observed, as well as instability on cooling (Fig. A1 -Supporting Information), indication that during heating there is a change in the system Cellulose/TMG that could not be reverted during the cooling step. A plausible reason (Roy et al., 2003) for such behavior is the increment of cellulose interchain hydrophobic interactions, which are not reversible on cooling, and/or degradation of cellulose during heating.

The data presented in Fig. 4, steady shear flow with temperature for the three concentrations of cellulose in TMG, indicates that there is proportional increase of viscosity with cellulose concentration. At lower concentrations, a viscosity plateau can be seen up to $50^{\circ} \mathrm{C}$, followed by a slight decrease. At $1 / 1 \mathrm{Cel} / \mathrm{TMG}$ concentration, the viscosity plateau region is not apparent and the slope of the viscosity decrease with temperature is almost constant. The decrease of viscosity with the increment of temperature is expected for systems where the hydrogen bond-based interactions become progressively weaker. 


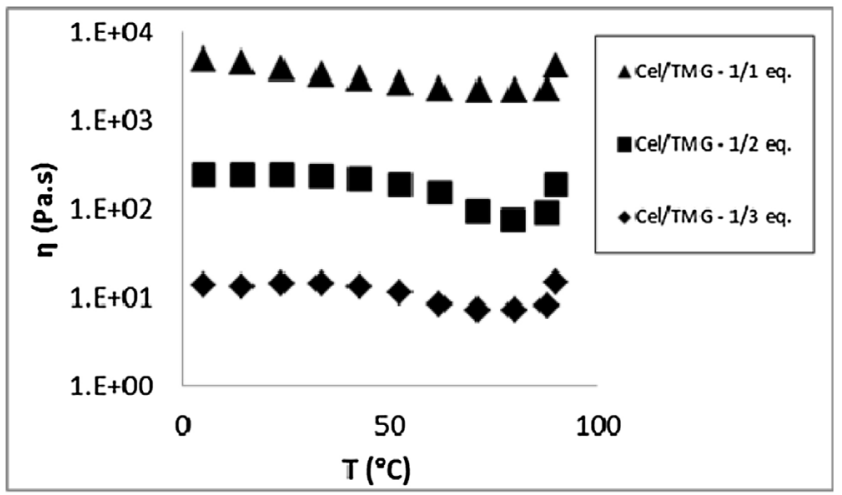

Fig. 4. $\eta$ (viscosity) vs $T$ (temperature). $\Upsilon$ (shear rate) $=1 \mathrm{~s}^{-1}$, three concentrations of cellulose (Cel) in tetramethylguanidine (TMG).

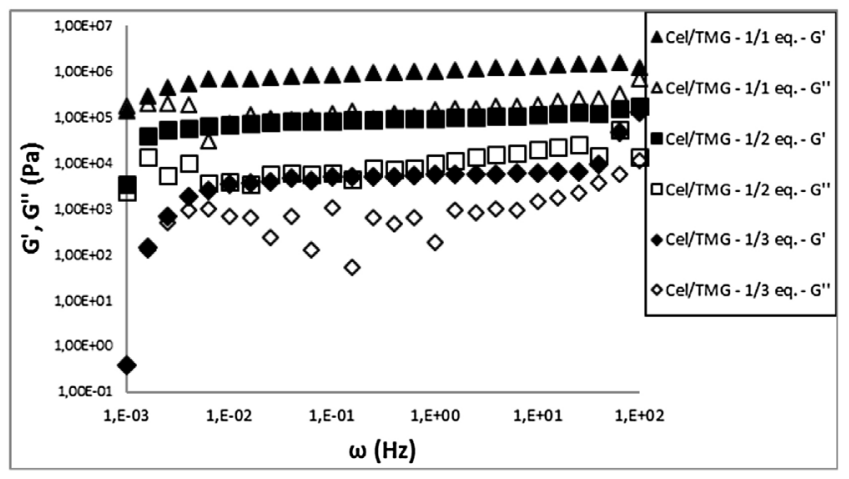

Fig. 5. $\mathrm{G}^{\prime}$ (storage moduli) and $\mathrm{G}^{\prime \prime}$ (loss moduli) vs $\omega$ (frequency) in $\mathrm{Hz}, \mathrm{T}=5{ }^{\circ} \mathrm{C}$ for all concentrations of cellulose (Cel) in tetramethylguanidine (TMG) as solvent matrix. $\tau=0.1 \mathrm{~Pa}$ (Cel/TMG: $1 / 3$ eq.), $\tau=10 \mathrm{~Pa}$ (Cel/TMG: $1 / 2$ eq.), $\tau=100 \mathrm{~Pa}$ (Cel/TMG: $1 / 1$ eq.).

For all concentrations studied, a sudden steep viscosity overshoot around $90^{\circ} \mathrm{C}$ was observed, caused by a gel matrix contraction with syneresis, i.e, phase separation.

The viscosity data of Fig. 4 gives a strong indication that intermediate temperatures could be optimal for an application of these materials as solutions, films and membranes for $\mathrm{CO}_{2}$ capture. Low viscosities and temperatures compatible with $\mathrm{CO}_{2}$ inclusion in the gels can be a good criterion of selection. An additional study (Fig. 5) represents the mechanical spectra, i.e., the viscoelastic properties $\mathrm{G}^{\prime}$ and $\mathrm{G}^{\prime \prime}$ as a function of the frequency, for the three concentrations of cellulose in TMG solvent matrix. It can be observed that, $G^{\prime}$ is higher than $G^{\prime \prime}$, and both are weakly dependent on frequency. In the zone where $G^{\prime}$ and $G^{\prime \prime}$ are practically constant, there is a difference of approximately one decade between $\mathrm{G}^{\prime}$ and $\mathrm{G}^{\prime \prime}$ and a minimum on $\tan \delta\left(G^{\prime \prime} / G^{\prime}\right)$. This is usual in complex flow structured systems, like emulsions stabilized by proteins (Raymundo, Franco, Empis \& Sousa, 2002) or gels from biopolymer mixtures. (Batista et al., 2011; Nunes, Raymundo \& Sousa, 2006; Shogren, Peterson, Evans \& Kenar, 2011)

This is the typical behavior of weak but structured gels (Fig. A2 -Supporting information and Ikeda \& Nishinari, 2001). As expected for these systems, the values of $G^{\prime}$ and $G^{\prime \prime}$ increase with the increment of the concentration of the polymer, in this case, cellulose.

The high structuration observed at mid-range frequencies, shorter times, can be explained by the non-disentanglement between cellulose chains detected during the short period of oscillation, corresponding to a second level of physical entanglements (Ferry, 1980). Differently, at low frequencies, there is sufficient time for the entanglement dynamics to shift to net disentanglement, giving a similar response to non-entangled matrices (Angioloni et al.,
2009). Moreover, in Fig. A3 (Supporting Information), a steady shear flow test (viscosity vs shear rate) is presented, where the shear thinning behaviour (decrease of viscosity with shear rate) is indicative of non-newtonian fluids (for the considered concentrations) in a similar way as aqueous cellulose systems previously studied (Lue et al., 2009).

\subsection{Spectroscopic and additional characterization}

In addition to rheological characterization, complete spectroscopic and microscopic SEM studies were carried out.

\subsubsection{Thermal ${ }^{1} H$ NMR}

The hydrogen bonds between cellulose and TMG become weaker with the increase of temperature according to the results of Fig. 6 (thermal ${ }^{1} \mathrm{H}$ NMR spectra). Specifically it is represented, on the left side of Fig. 6, the hydrogen bond-based $\mathrm{OH}-\mathrm{NH}$ protons from cellulose and TMG, respectively. This type of band is placed at low field where the distinction between the different $-\mathrm{OH}$ protons from cellulose and $\mathrm{NH}$ proton from TMG vanishes. This situation can be explained by the combination of two effects: extended hydrogen bond network (Carrera, Costa, Nunes da Ponte \& Branco, 2013) and the increased rate of exchange of the protons in the hydroxyl functionalities. (Silverstein \& Webster, 1997) According to Fig. 6, the displacement of this band to a higher field with the increase of temperature is a clear indication of weakening of the hydrogen bond interactions (Carrera et al., 2013) between cellulose and TMG. The $-\mathrm{CH}_{3}$ protons of TMG are represented on the right side of Fig. 6. Unexpectedly at first sight, they are also sensitive to temperature. Accordingly, the band at $2.3 \mathrm{ppm}$ is shifted to lower field at higher temperatures. To explain this effect, it is important to highlight the work of (Vitorino et al., 2014) on the crystallographic structure of tetramethylguanidinium nitrate. On the two polymorphs found, a hydrogen bond based interaction $\mathrm{HN}-\mathrm{H}-$ $\mathrm{ONO}_{2}$ between tetramethylguanidinium and nitrate is clear, similar to the HN-HO interaction between TMG and cellulose. Additionally, both polymorphs in Vitorino et al. present another type of interaction: $\mathrm{H}_{2} \mathrm{C}-\mathrm{H}-\mathrm{ONO}_{2}$ between the methyl group of tetramethylguanidinium and nitrate. Considering this last aspect, a very reliable explanation for the effect found on Fig. 6 for the $\mathrm{CH}_{3}$ protons of TMG is based on a $\mathrm{H}_{2} \mathrm{C}-\mathrm{H}-\mathrm{OHC}$ interaction between methyl protons of TMG and unshared oxygen electrons of the alcohol groups from cellulose. At higher temperatures, such interactions become weaker and, progressively, the methyl protons become unshielded. These results are in line with previously reported behavior of cellulose system with increasing temperature. (Zhang, Li, Yuc \& Hsieh, 2010)

Data on Figs. 3 and 6 give a strong indication of entanglements between cellulose chains and, simultaneously, a weakening of hydrogen bond-based interactions between cellulose and TMG with increase of temperature.

\subsubsection{SEM}

The SEM images of the prepared gels of cellulose are represented in Fig. 7. Going from the more diluted to the more concentrated gel (a, b, c), there is an increased homogeneity (less irregularities on the surface). This is an indication of a more effective cluster formation between the elements of the gel network, leading to an increased viscosity, as evident in Fig. 4, comparing different concentrations at the same temperature, and in line with the work of (Roy et al., 2003).

\subsubsection{Crystalline index of cellulose isolated from a selected gel}

Carbon nuclear magnetic resonance $\left({ }^{13} \mathrm{C}\right.$-CPMAS-NMR) and $\mathrm{X}$ ray diffraction (XRD) studies were carried out in order to compare 

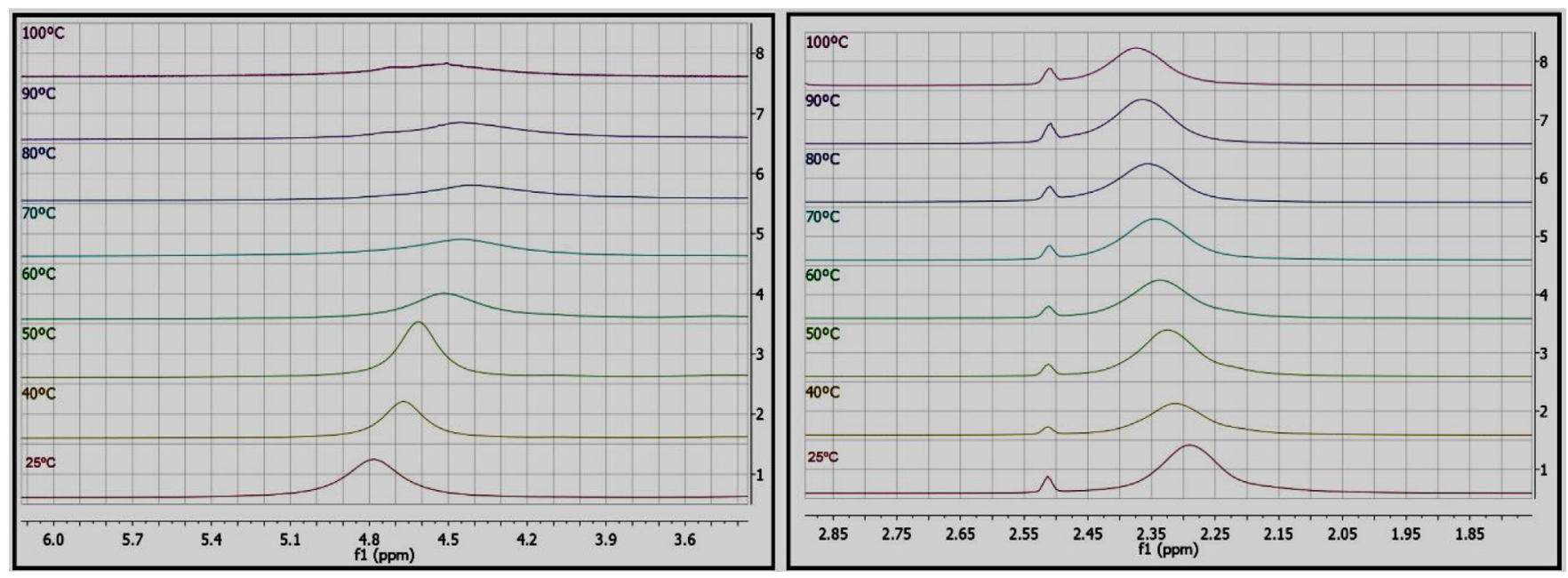

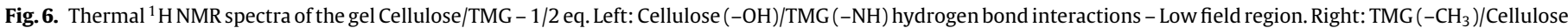
$(-\mathrm{OH})$ interactions - High field region.
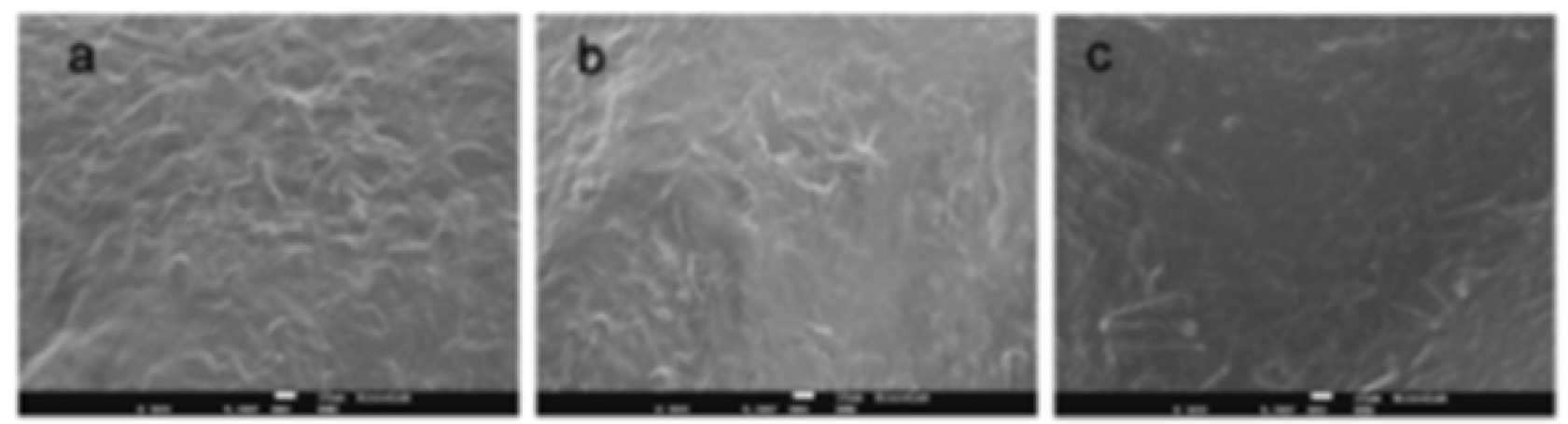

Fig. 7. a, b, c - SEM images (500x) of gels of cellulose/TMG - 1/3,1/2,1/1 ratios in equivalents respectively.

the crystalline index $(\mathrm{CI})$ of the cellulose recovered from the intermediate concentration gel $1 / 2$ (see Materials and methods) with the index of commercial microcrystalline cellulose. Decreasing of the crystallinity facilitates chemical modification, and it would therefore be a favorable result in the context of possible applications.

3.2.3.1. ${ }^{13} \mathrm{C}$-CPMAS-NMR. It is possible to observe, by ${ }^{13} \mathrm{C}$-CPMASNMR (Fig. 8), that the isolated cellulose and commercial cellulose samples have similar spectral profiles, with characteristic peaks associated to each specific carbon of repetitive unit of cellulose. According to (Xia et al., 2015), it is possible to estimate the CI of cellulose by comparing the areas of the two bands associated to the C4 carbon, with the band at lower field corresponding to the crystalline phase, and with the other, at higher field, corresponding to an amorphous phase. The results obtained in this work are shown in Fig. 8, where it is apparent that the ratio between the areas of the crystalline phase and the amorphous phase bands decrease significantly from the commercial sample to the cellulose isolated in this work. Using these area ratios as a measure, they indicate $51 \%$ crystallinity for the commercial microcrystalline cellulose and a CI of $32 \%$, for the cellulose sample after gelification with TMG and coagulation with ethanol.

3.2.3.2. XRD measurements. The analysis of the XRD spectra of the samples followed the method of (Segal, Creely, Martin \& Conrad, 1959). This method was applied more recently by other authors (Nam, French, Condon \& Concha, 2016). It yielded a crystalline index of $93 \%$ for the commercial microcrystalline cellulose and of
$8 \%$ for the cellulose obtained in this work, after formation of gel and isolation using ethanol.

Although the results from the two methods are very different, they follow, nevertheless, the same trend of clearly decreased crystallinity of the cellulose obtained in this work. In fact, (Park, Baker, Himmel, Parilla \& Johnson, 2010) have already highlighted the discrepancies between the two methods for commercial Avicel PH-101 cellulose, where a value of $91.7 \%$ was obtained with Seagal (height) XRD method, contrasting with $56.7 \%$ of CI obtained by ${ }^{13} \mathrm{C}$-CPMAS-NMR, similarly as in the instance of this work.

\subsubsection{Aqueous colloidal mixtures}

In a different perspective, water was added $(3.5 \mathrm{~mL})$ to the gel of intermediate concentration of cellulose (Fig. A4 - A - Supporting Information), forming a stable colloidal mixture during $48 \mathrm{~h}$ at $11^{\circ} \mathrm{C}$. In order to check the effect of the superbase for the observed behavior a similar test was carried out, but excluding TMG (Fig. A4 B - Supporting Information). After $40 \mathrm{~min}$ at $11^{\circ} \mathrm{C}$, cellulose precipitated. This is a clear indication of the effect of TMG in promoting the dispersion of cellulose among water based colloid. The effect of the low temperature $\left(11^{\circ} \mathrm{C}\right)$ in promoting long-lasting stability of the Cel/TMG aqueous colloid in opposition to similar experiment carried out at room temperature (start precipitation after $4 \mathrm{~h}$ at $23^{\circ} \mathrm{C}$ ) shows that hydrogen bond-based interactions are responsible for the stabilization of cellulose in aqueous media.

The possibility to have cellulose dispersed and more intimately interacting with the water as solvent is a step forward in design- 


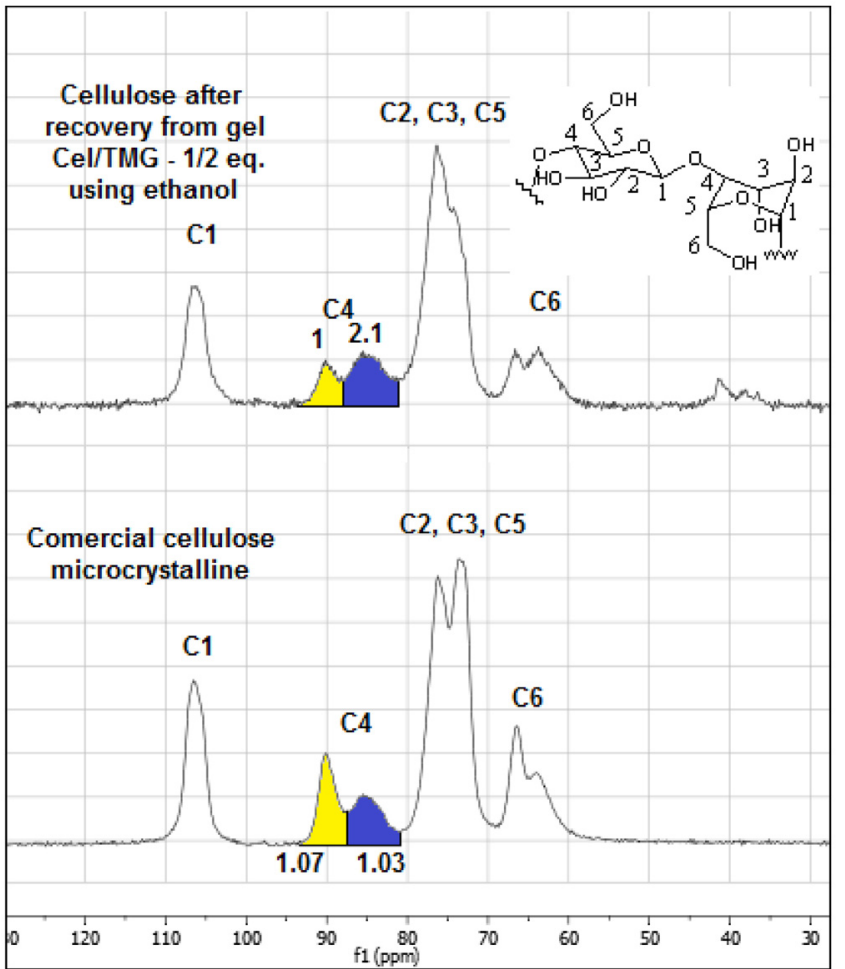

Fig. 8. ${ }^{13} \mathrm{C}$-CPMAS-NMR spectra of microcrystalline cellulose previous and after formation of gel with TMG and recovery using ethanol.

ing reacting systems for chemical and physical modification of this polymer.

\section{Conclusions}

The reported work features the generation of gels of cellulose based on tetramethylguanidine. As far as we know, the result of $46.5 \mathrm{wt} \%$ obtained in this work is the maximum uptake of cellulose by a solvent reported so far. These systems may find application in the field of physical/chemical modification of cellulose and as bulk mixtures, films and membranes for $\mathrm{CO}_{2}$ capture. Regarding this last aspect, the abundance of cellulose and the low cost of TMG constitute clear advantages in the use of these gels as $\mathrm{CO}_{2}$ capture agents. The increase of temperature led to weakening of the diverse hydrogen bond interactions between cellulose and TMG, corresponding to a significant decrease of viscosity. Simultaneously, the entanglement between the different chains of cellulose increase with temperature, corresponding to sol-gel transition and structuration of the gels. The transition temperature presents an opposite trend inherently to the increase of concentration of cellulose in the gel. Furthermore, rheological characterization shows that these are weak but well-structured gels. Using ethanol to recover cellulose from the gel leads to a significant decrease of cellulose crystallinity, with possible implications in further processing of this polymer. Finally adding water as solvent to a cellulose gel-based system leads to a stable colloid. This result may be seen as a step forward in the design of reacting systems for chemical modification of this polymer.

\section{Acknowledgements}

This work was supported by Fundação para a Ciência e Tecnologia (PEst-C/EQB/LA0006/2013) and a postdoctoral fellowship GVSMC - (SFRH/BPD/72095/2010), project (UID/CTM/50025/2013) and COMPETE program. The authors acknowledge: Dr. Marta Corvo from CENIMAT (FCT/UNL) and Dr. Isabel Nogueira (MicroLabLAboratório de Microscopia Electrónica do Instituto Superior Técnico), for recording and edition of ${ }^{13} \mathrm{C}-\mathrm{SS}$-CPMAS-NMR spectra and recording of SEM images, respectively. Dr. César Laia and Prof. Madalena Dionisio are acknowledged for fruitful discussions. The NMR spectrometers are part of The National NMR Facility, supported by Fundação para a Ciência e a Tecnologia (RECI/BBBBQB/0230/2012).

\section{Appendix A. Supplementary data}

Supplementary data associated with this article can be found, in the online version, at http://dx.doi.org/10.1016/j.carbpol.2017.03. 084 .

\section{References}

Angioloni, A., \& Collar, C. (2009). Small and large deformation viscoelastic behaviour of selected fibre blends with gelling properties. Food Hydrocolloids, 23, 742-748.

Batista, A. P., Nunes, M. C., Raymundo, A., Gouveia, L., Sousa, I., Cordobés, F., et al. (2011). Microalgae biomass interaction in biopolymer gelled systems. Food Hydrocolloids, 25, 817-825.

Burchard, W. (2001). Structure formation by polysaccharides in concentrated solution. Biomacromolecules, 2, 342-353.

Cai, J., \& Zhang, L. (2006). Unique gelation behavior of cellulose in $\mathrm{NaOH} / \mathrm{Urea}$ aqueous solution. Biomacromolecules, 7, 183-189.

Carrera, G. V. S. M., Nunes da Ponte, M., \& Branco, L. C. (2012). Synthesis and properties of reversible ionic liquids using $\mathrm{CO}_{2}$, mono- to multiple functionalization. Tetrahedron, 68, 7408-7413.

Carrera, G. V. S. M., Costa, A., Nunes da Ponte, M., \& Branco, L. C. (2013). Use of organic superbases and temperature effects for the development of reversible protic amino acid salts. Synlett, 24, 2525-2530.

Carrera, G. V. S. M., Jordão, N., Branco, L. C., \& Nunes da Ponte, M. (2015a). CO capture and reversible release using mono-saccharides and an organic superbase. Journal of Supercritical Fluids, 105, 151-157.

Carrera, G. V. S. M., Jordão, N., Branco, L. C., \& Nunes da Ponte, M. (2015b). CO 2 capture systems based on saccharides and organic superbases. Faraday Discussions, 183, 429-444.

Carrera, G. V. S. M., Jordão, N., Santos, M. M., Nunes da Ponte, M., \& Branco, L. C. (2015). Reversible systems based on $\mathrm{CO}_{2}$, amino-acids and organic superbases. RSC Advances, 5, 35564-35571.

Chambon, F., \& Winter, H. H. (1987). Linear viscoelasticity at the gel point of a crosslinking PDMS with imbalanced stoichiometry. Journal of Rheology, 31(8), 683-697.

Ferry, J. D. (1980). Viscoelastic properties of polymers (3rd ed.). John Wiley., p. 366

Frey, M. W., Chan, H., \& Carranco, K. (2005). Rheology of cellulose/KSCN/ethylenediamine solutions and coagulation into filaments and films. Journal of Polymer Science.: Part B: Polymer Physics, 43, 2013-2022.

Gavillon, R., \& Budtova, T. (2007). Kinetics of cellulose regeneration from cellulose- $\mathrm{NaOH}$-water gels and comparison with cellulose- $N$-methylmorpholine- $N$-oxide-water solutions. Biomacromolecules, $8,424-432$

Ikeda, S., \& Nishinari, K. (2001). Weak gel-type rheological properties of aqueous dispersions of nonaggregated $\kappa$-carrageenan helices. Journal of Agricultural and Food Chemistry, 49, 4436-4441.

King, A. W. T., Asikkala, J., Mutikainen, I., Järvi, P., \& Kilpeläinen, I. (2011). Distillable acid--base conjugate ionic liquids for cellulose dissolution and processing. Angewandte Chemie, 123, 6425-6429.

Li, L., Thangamathesvaran, P. M., Yue, C. Y., Tam, K. C., Hu, X., \& Lam, Y. C. (2001). Gel network structure of methylcellulose in water. Langmuir, 17, 8062-8068.

Lue, A., \& Zhang, L. (2009). Rheological behaviors in the regimes from dilute to concentrated in cellulose solutions dissolved at low temperature. Macromolecule Bioscience, 9, 488-496.

Nam, S., French, A. D., Condon, B. D., \& Concha, M. (2016). Segal crystallinity index revisited by the simulation of X-ray diffraction patterns of cotton cellulose I and cellulose II. Carbohydrate Polymers, 135, 1-9.

Nunes, M. C., Raymundo, A., \& Sousa, I. (2006). Rheological behaviour and microstructure of pea protein/k-carrageenan/starch gels with different setting conditions. Food Hydrocolloids, 20, 106-113.

Park, S., Baker, J. O., Himmel, M. E., Parilla, P. A., \& Johnson, D. K. (2010). Cellulose crystallinity index: Measurement techniques and their impact on interpreting cellulase performance. Biotechnology for Biofuels, 3, 10.

Qin, X., Lu, A., \& Zhang, L. (2012). Effect of stirring conditions on cellulose dissolution in $\mathrm{NaOH} /$ urea aqueous solution at low temperature. Journal of Applied Polymer Science, 126, E469-E476.

Raymundo, A., Franco, J. M., Empis, J., \& Sousa, I. (2002). Optimization of the composition of low-fat oil-in-water emulsions stabilized by white lupin protein. Journal of American Oil Chemical Society, 79, 783-790.

Roy, C., Budtova, T., \& Navard, P. (2003). Rheological properties and gelation of aqueous cellulose- $\mathrm{NaOH}$ solutions. Biomacromolecules, 4, 259-264. 
Ruan, D., Lue, A., \& Zhang, L. (2008). Gelation behaviors of cellulose solution dissolved in aqueous $\mathrm{NaOH} /$ thiourea at low temperature. Polymer, 49 , 1027-1036.

Segal, L., Creely, J. J., Martin, A. E., \& Conrad, C. M. (1959). An empirical method for estimating the degree of crystallinity of native cellulose using the $\mathrm{X}$-ray diffractometer. Textile Research Journal, 29, 786-794.

Shogren, R. L. Peterson, S. C., Evans, K. O., \& Kenar, J. A. (2011). Preparation and characterization of cellulose gels from corn cobs. Carbohydrate Polymers, 86, 1351-1357.

Silverstein, R. M., \& Webster, F. X. (1997). Spectrometric identification of organic compounds. pp. 163-168. New York: Jonh Wiley \& Sons, Inc.

Tanaka, T. (1981). Gels. Scientific American, 244, 124-138.

Vitorino, J., Agapito, F., Piedade, M. F. M., Bernardes, C. E. S., Diogo, H. P., Leal, J. P., et al. (2014). Thermochemistry of 1,1,3,3-tetramethylguanidine and

1,1,3,3-tetramethylguanidinium nitrate. The Journal of Chemical Thermodynamics, 77, 179-189.

Wang, H., Gurau, G., \& Rogers, R. D. (2012). Ionic liquid processing of cellulose. Chemical Society Reviews, 41, 1519-1537.
Wang, J., Xue, Z., Yan, C., Li, Z., \& Mu, T. (2016). Fine regulation of cellulose dissolution and regeneration by low pressure $\mathrm{CO}_{2}$ in DMSO/organic base: Dissolution behavior and mechanism. Physcal Chemistry Chemical Physics, 18 32772-32779.

Xia, Z., Patchan, M., Maranchi, J., \& Trexler, M. (2015). Structure and relaxation in cellulose hydrogels. Journal of Applied Polymer Science, $132 \mathrm{http}$ //dx.doi.org/10. 1002/app.42071.0

Zhang, S., Li, F.-X., Yuc, J.-Y., \& Hsieh, Y.-L. (2010). Dissolution behaviour and solubility of cellulose in NaOH complex solution. Carbohydrate Polymers, 81, 668-674.

Zhang, M., Ding, C., Huang, L., Chen, L., \& Yang, H. (2014). Interactions of collagen and cellulose in their blends with 1-ethyl-3-methylimidazolium acetate as solvent. Cellulose, 21, 3311-3322.

Zhou, J., Zhang, L., Deng, Q. \& Wu, X. J. (2004). Synthesis and characterization of cellulose derivatives prepared in $\mathrm{NaOH} /$ urea aqueous solutions. Journal of Polymer Science: Part A: Polymer Chemistry, 42, 5911-5920. 